\title{
Iterative Multiuser Detection and Channel Decoding for DS-CDMA Using Harmony Search
}

\author{
Rong Zhang and Lajos Hanzo, Fellow, IEEE
}

\begin{abstract}
A novel random-guided optimization method is proposed for multiuser detection (MUD) in DS-CDMA systems employing the so-called Harmony Search (HS) algorithm. We specifically design the HS-aided MUD for the communications problem considered and apply it in an iterative joint MUD and channel decoding framework. Our simulation results demonstrate that a near-single-user performance can be achieved without the employment of the full-search-based optimum MUD even in extremely highly loaded DS-CDMA systems.
\end{abstract}

Index Terms-Evolutionary Algorithm, , Harmony Search Algorithm, iterative receiver, Markov Chain Monte Carlo Algorithm, multiuser detection, .

\section{INTRODUCTION}

I $\mathrm{N}$ the context of iterative detection and decoding (IDD) [1] aided multiuser detection (MUD), stochastic global optimization techniques may be used in order to reduce the complexity, while still capturing the optimum full-search-based solution with a high probability using, for example, the Genetic Algorithm (GA) [2] or the Metropolis-Hastings (MH) type algorithm [3].

Imitating the improvisation process of musicians, a new meta-heuristic optimisation method referred to as the Harmony Search (HS) algorithm was proposed in [4]. When a musician improvises, the aesthetic value referred to as the fitness function $(\mathrm{FF})$ is determined by a set of pitches produced by the music instruments (variables) involved. The musician seeks to produce aesthetically pleasing harmony (the optimum solution) as determined by his/her aesthetic perception inferred from rehearsals (iterations).

In this paper, we design a HS-aided MUD algorithm, which is capable of approaching the optimum MUD's performance with low complexity.

The remainder of this paper is organized as follows. In Section II, we introduce the system model and specifically design the HS algorithm in the context of IDD in Section III. We

Manuscript received April 20, 2009; revised June 13, 2009. First published July 10, 2009; current version published August 12, 2009. This work was supported by EPSRC as part of the Core 4 Research Programme of the Virtual Center of Excellence in Mobile and Personal Communications, Mobile VCE, www.mobilevce.com. The associate editor coordinating the review of this manuscript and approving it for publication was Prof. Tongtong $\mathrm{Li}$.

The authors are with the School of Electrical and Computer Science, University of Southampton, Southampton SO17 1BJ, U.K. (e-mail: lh@ecs.soton.ac.uk; http://www-mobile.ecs.soton.ac.uk).

Color versions of one or more of the figures in this paper are available online at http://ieeexplore.ieee.org.

Digital Object Identifier 10.1109/LSP.2009.2027159 then characterize its performance in Section IV, and conclude in Section V.

\section{System Model AND PRoBlem Formulation}

In the context of the IDD scheme seen in Fig. 1, we consider a rate- $R$ coded BPSK modulated $K$-user DS-CDMA system employing user-specific $N_{c}$-chip random spreading sequences. The assumption of a long interleaver within the IDD allows us to focus on a particular symbol interval $\mathbf{y}=\mathbf{C H x}+\mathbf{n}$, where $\mathbf{y} \in \mathcal{C}^{N_{c} \times 1}, \mathbf{x} \in \mathcal{R}^{K \times 1}$, and $\mathbf{C} \in \mathcal{R}^{N_{c} \times K}$ are the received sample vector, the transmitted symbol vector, and the DS-CDMA spreading matrix, respectively. Furthermore, $\mathbf{n} \in$ $\mathcal{C}^{N_{c} \times 1}, n_{i} \sim \mathcal{C N}\left(0, \sigma^{2}\right)$ is the complex additive white Gaussian noise (AWGN) vector and $\mathbf{H}=\operatorname{diag}\left[h_{1}, \ldots, h_{k}\right]$ represents the block-invariant complex channel.

After observing the a priori information in terms of log-likelihood ratios (LLRs) denoted by $\mathcal{L}^{a}(\mathbf{x})$, the extrinsic information $\mathcal{L}^{e}(\mathbf{x})$ is delivered by the MUD to the outer channel decoder. Based on the independence of each user's information, the $e x$ trinsic LLR of the $k$ th user is given by $\mathcal{L}^{e}\left(x_{k}\right)=\mathcal{L}\left(x_{k}\right)-$ $\mathcal{L}^{a}\left(x_{k}\right)$, where the a posteriori $\operatorname{LLR} \mathcal{L}\left(x_{k}\right)$ is

$$
\begin{aligned}
\mathcal{L}\left(x_{k}\right) & =\ln \frac{P\left[x_{k}=+1 \mid \mathbf{y}, \mathcal{L}^{a}(\mathbf{x})\right]}{P\left[x_{k}=-1 \mid \mathbf{y}, \mathcal{L}^{a}(\mathbf{x})\right]} \\
& =\ln \frac{\sum_{\forall \mathbf{x}_{-k}} P\left[x_{k}=+1, \mathbf{x}_{-k} \mid \mathbf{y}, \mathcal{L}^{a}(\mathbf{x})\right]}{\sum_{\forall \mathbf{x}_{-k}} P\left[x_{k}=-1, \mathbf{x}_{-k} \mid \mathbf{y}, \mathcal{L}^{a}(\mathbf{x})\right]}
\end{aligned}
$$

where $\mathbf{x}_{-k}$ denotes the $K$-user vector with the $k$ th element $x_{k}$ excluded. We note that all possible $2^{K-1}$ vectors are required in the above summation, which leads to a prohibitive complexity. This motivates the development of a range of low-complexity MUD detection algorithms, such as those in [2] and [5] and the proposed HS algorithm of the next section.

\section{HARMONY SEARCH AIDED ITERATIVE RECEIVER}

We approximate the summation of all legitimate $K$-user vectors in terms of the Bayesian optimum of (1) by collecting a sufficient number of significant $K$-user candidate vectors $\mathbf{x}$, where the 'collection' is followed by a set of HS rules and the "significance" is quantified by the FF to be introduced below.

\section{A. Fitness Function of Harmony Search Aided MUD}

We define the FF to be evaluated as the joint a posteriori probability (APP) of the $K$-user transmitted vector $\mathbf{x}$ based on the observation $\mathbf{y}$ and the a priori LLRs $\mathcal{L}^{a}(\mathbf{x})$ provided by the channel decoder, which may be expressed as 


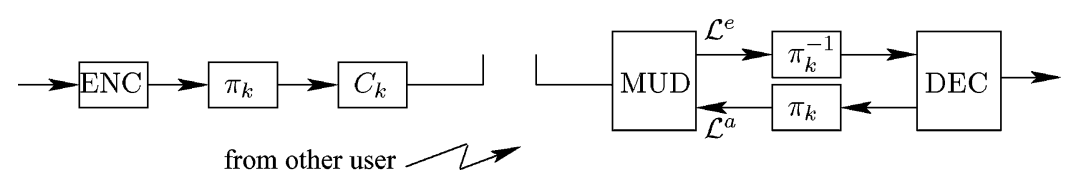

Fig. 1. Iterative multiuser detection and channel decoding, where ENC is short for encoding.

$$
\begin{aligned}
f(\mathbf{x}) & =\ln P\left[\mathbf{x} \mid \mathbf{y}, \mathcal{L}^{a}(\mathbf{x})\right] \\
& \propto \ln p(\mathbf{y} \mid \mathbf{x}) P\left[\mathbf{x} \mid \mathcal{L}^{a}(\mathbf{x})\right] \\
& \propto \frac{-\|\mathbf{y}-\mathbf{C H x}\|^{2}}{2 \sigma^{2}}+\sum_{\forall k} \ln P^{a}\left(x_{k}\right)
\end{aligned}
$$

where $P^{a}\left(x_{k}\right)=P\left[x_{k} \mid \mathcal{L}^{a}\left(x_{k}\right)\right]$ denotes the a priori probability of each user $x_{k}$, which may be expressed as $P^{a}\left(x_{k}=+1\right)=$ $1 /\left[1+e^{-\mathcal{L}^{a}\left(x_{k}\right)}\right]$.

\section{B. Naive Transplanting of the Harmony Search Algorithm}

We first introduce a range of parameters associated with the original HS algorithm [4]. The harmony memory size $M$ specifies the size of the harmony memory matrix (HMM), which hosts the number of initial $K$-user harmony candidates. The harmony memory activation probability $P_{m a}$ specifies the probability of generating a new $K$-user candidate from the HMM, rather than being randomly selected with a probability of $(1-$ $\left.P_{m a}\right)$. The pitch adjustment probability $P_{p a}$ specifies the probability of further tuning the newly generated $K$-user candidate from the HMM with a probability of $P_{m a}$. Finally, $Q$ represents the total number of improvisations executed. Explicitly, the HS algorithm can be summarized as follows.

Initialization: We initialize the HMM $\mathbf{X}^{0}$ hosting $M$ randomly generated $K$-user candidate harmony vectors $\mathbf{X}^{0}=\left[\mathbf{x}_{1}^{0}, \ldots \mathbf{x}_{M}^{0}\right]$ from the set of $2^{K}$ legitimate solutions.

Improvisation: Three HS operations are applied during each improvisation, namely the memory activation, pitch adjustment and random selection. In particular, a new $K$-user harmony vector $\mathrm{x}^{\text {new }}$ may be randomly selected from the alphabet $x_{k}^{\text {new }} \in \mathcal{A}_{k}=\{ \pm 1\}$ with a probability of $\left(1-P_{m a}\right)$ or selected from the HMM $\mathbf{X}^{q-1}$ with a probability of $P_{m a}$ according to $x_{k}^{\text {new }}=x_{m_{i}, k}^{q-1}, m_{i} \sim \mathcal{U}[1, M], k \in[1, K]$, which implies inheriting the $k$ th bit of one of the $M$ candidate vectors in the $(q-1)$ th iteration $k \in[1, K]$. Once the memory activation was carried out, a further pitch adjustment characterised by a step of $\Delta$ may be applied with a pitch adjustment probability of $P_{p a}$. In detail, the specific value of each variable $x_{k}^{\text {new }}$, $k \in[1, K]$ of the new $K$-user harmony candidate is tuned to match the neighboring values in its legitimate solution alphabet $\mathcal{A}_{k}=\{ \pm 1\}$.

Updating: The harmony vector having the worst $\mathrm{FF}$ in $\mathbf{X}^{q-1}$ is replaced by the newly generated $K$-user harmony vector, provided it is less fit than the new one, otherwise it survives for the next iteration.

\section{Pitch Adjustment in the Harmony Search Aided MUD}

In the pitch adjustment step, the direct employment of the HS algorithm would entail binary toggling of the related bit $x_{k}^{\text {new }} \in$
$\{ \pm 1\}, k \in[1, K]$ based on the predefined probability of $P_{p a}$ and hence may lead to a dramatically different $K$-user counterpart, which would be disadvantageous in our MUD. Instead, we thus propose to take the soft information associated with $x_{k}^{\text {new }}$ into account for determining $P_{p a}$. As a result, the specific value of $x_{k}^{\text {new }}$ will be altered during each pitch adjustment step based on the probability representing the related soft information.

At iteration $q$, after randomly selecting a $K$-user base harmony vector $\mathbf{x}^{b}$ from the HMM X $\mathbf{X}^{q-1}$ with a probability of $P_{m a}$, the pitch adjustment is carried out by generating the $k$ th variable $x_{k}^{\text {new }}$ based on the marginal APP $P\left[x_{k}^{b} \mid \mathbf{y}, \mathbf{x}_{-k}^{b}, \mathcal{L}^{a}\left(x_{k}\right)\right]$ of the base harmony vector, where $\mathbf{x}_{-k}^{b}$ denotes the $K$-user base vector with the $k$ th element $x_{k}^{b}$ excluded. This automatically updated marginal APP represents the soft information of $x_{k}^{\text {new }}$ and acts as the replacement of $P_{p a}$ in the original HS proposal of [4]. This reflects the process of musical improvisation, where a particular instrument adjusts its pitch based on the harmony generated by other instruments. The LLR of this marginal APP may be conveniently evaluated in the following way:

$$
\begin{aligned}
\mathcal{L}_{p a} & =\ln \frac{P\left[x_{k}^{b}=+1 \mid \mathbf{y}, \mathbf{x}_{-k}^{b}, \mathcal{L}^{a}\left(x_{k}\right)\right]}{P\left[x_{k}^{b}=-1 \mid \mathbf{y}, \mathbf{x}_{-k}^{b}, \mathcal{L}^{a}\left(x_{k}\right)\right]} \\
& =\ln \frac{p\left(\mathbf{y} \mid x_{k}^{b}=+1, \mathbf{x}_{-k}^{b}\right)}{p\left(\mathbf{y} \mid x_{k}^{b}=-1, \mathbf{x}_{-k}^{b}\right)}+\mathcal{L}^{a}\left(x_{k}\right) \\
& =\frac{\left\|\mathbf{y}-\mathbf{C H x}_{k-}^{b}\right\|^{2}-\left\|\mathbf{y}-\mathbf{C H} \mathbf{x}_{k+}^{b}\right\|^{2}}{2 \sigma^{2}}+\mathcal{L}^{a}\left(x_{k}\right)
\end{aligned}
$$

where the subscript $+/-$ indicates the sign of the $k$ th element $x_{k}^{b}$ of the $K$-user base vector. Hence, we have

$$
P_{p a}\left(x_{k}^{\text {new }}=+1\right)=\frac{1}{1+e^{-\mathcal{L}_{p a}}} .
$$

In this case, the $P_{m a}$-based memory activation and the $P_{p a}$-based pitch adjustment merge into a single joint step. The marginal APP-based pitch adjustment of (3) is capable of providing a sufficiently high decision reliability, hence the magnitude of the LLRs is improved during the successive improvisations. This implies that the selection of a new $K$-user harmony vector for inclusion in the HMM is more appropriate than a random choice. Hence, we may set the probability $P_{m a}=1$ and appropriately avoid the operation of random selection. In other words, artificially enforcing a random selection with a probability of $\left(1-P_{m a}\right)$ is not recommended, since the improvisations based on this random selection may be wasted. Importantly, ignoring the associated random selection does not limit the exploration capability of the HS-aided MUD, because if we have a sufficiently high $M$, randomly selected base vectors have first been generated, before fine-tuning the pitch adjustment. In summary, the pseudocode of our HS-aided MUD algorithm is shown in Table I. 
TABLE I

PSEUDOCODE OF THE HS-AIDED MUD

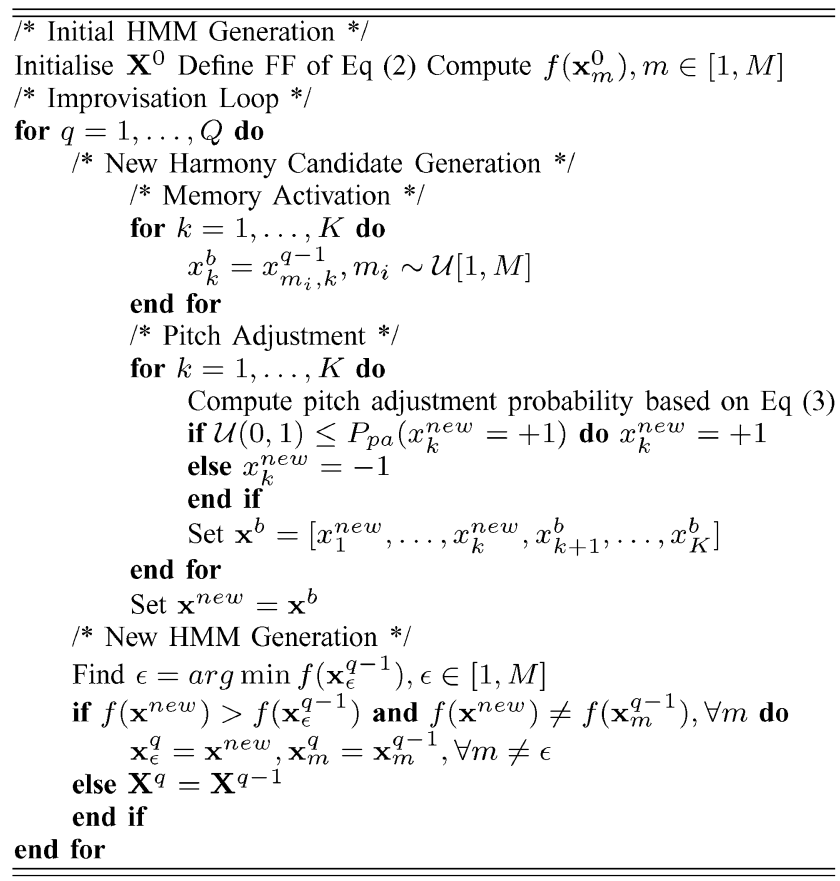

\section{Soft Output of the Harmony Search Aided MUD}

After $Q$ improvisations, we may approximate (1) based on the $M$ surviving $K$-user candidate vectors in the final HMM $\mathbf{X}^{Q}$, which can be expressed as

$$
\begin{aligned}
\mathcal{L}\left(x_{k}\right) & =\ln \frac{\sum_{\mathbf{x}_{-k} \in \mathbf{X}^{Q}} P\left[x_{k}=+1, \mathbf{x}_{-k} \mid \mathbf{y}, \mathcal{L}^{a}(\mathbf{x})\right]}{\sum_{\mathbf{x}_{-k} \in \mathbf{X}^{Q} P} P\left[x_{k}=-1, \mathbf{x}_{-k} \mid \mathbf{y}, \mathcal{L}^{a}(\mathbf{x})\right]} \\
& =\ln \sum_{\mathbf{x}_{-k} \in \mathbf{X}^{Q}} e^{f\left(\mathbf{x}_{k+}\right)}-\ln \sum_{\mathbf{x}_{-k} \in \mathbf{X}^{Q}} e^{f\left(\mathbf{x}_{k-}\right)}
\end{aligned}
$$

where $f\left(\mathbf{x}_{k+}\right)$ and $f\left(\mathbf{x}_{k-}\right)$ represent the FF function of a given candidate vector $\mathbf{x}$ having its $k$ th entry equals to +1 and -1 , respectively. When considering the extrinsic $\operatorname{LLR} \mathcal{L}^{e}\left(x_{k}\right)$, the corresponding extrinsic FF value $f^{e}(\mathbf{x})$ is substituted in (4), which may be given by $f^{e}(\mathbf{x})=f(\mathbf{x})-\ln P^{a}\left(x_{k}\right)$.

\section{PERFormance Evaluation}

\section{A. Parameters and Complexity}

Consider an outer rate $R=1 / 3$ repetition coded DS-CDMA system employing user-specific random spreading sequences of length $N_{c}=7$, where the information frame length was $N_{i}=512$ and the number of iterations between the MUD and the soft decoder was set to $I_{\mathrm{IDD}}=10$. Furthermore, we define the so-called normalised system-load $\beta=K / N_{c}$ as the ratio of the number of users supported to the spreading sequence length employed. An AWGN channel was assumed and a uniformly distributed transmission block-invariant channel phase noise was imposed, which was assumed perfectly known to the receiver.

Let us first discuss the complexity of our HS-aided MUD in terms of the required FF evaluations. Generating the a posteriori LLRs given by (1) for $K$ users requires $\mathcal{Q}_{\text {opt }}=K \times 2^{K}$ evaluations of (2), while the HS algorithm requires a total of

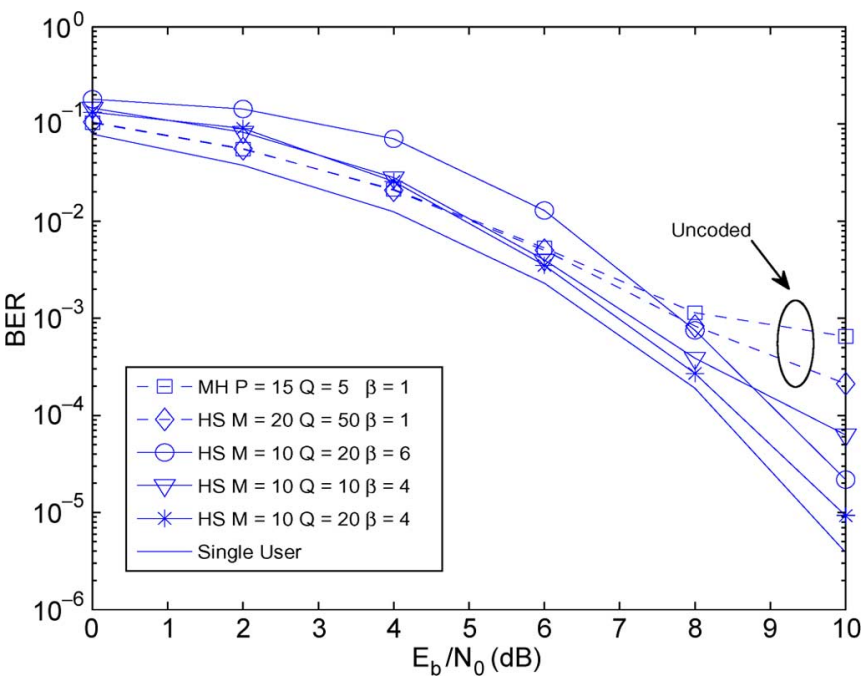

Fig. 2. BER performance of HS-aided MUD of a $R=1 / 3$-repetition coded BPSK modulated DS-CDMA system using $N_{c}=7$-chip random sequences and $I_{\mathrm{IDD}}=10$ iterations.

$$
\mathcal{Q}_{H S}=Q \times 2 \times K+Q+M+2 \times M \times K
$$

evaluations of the FF of (2), In detail, it includes $M$ FF evaluations of the initial HMM, and a further evaluation at the end of each of the $Q$ improvisations as well as $(2 \times K)$ evaluations, when generating the marginal APP based pitch adjustment of each of the $Q$ improvisations. In addition, the soft output generation requires a further $(2 \times M \times K)$ evaluations of (4).

\section{B. Simulation Results}

We first compare our HS-aided MUD to the conventional $\mathrm{MH}$ algorithm of a full-load uncoded DS-CDMA system. In this case, the soft output evaluation of (4) may be avoided by simply considering the best harmony vector in the final HMM, i.e. $\mathbf{x}^{*}=\max _{\mathbf{x} \in \mathbf{X}^{Q}} f(\mathbf{x})$, and as a result, the final additive term of (5) is neglected. Hence, our HS-aided MUD requires $Q_{H S}=770 \mathrm{FF}$ evaluations. On the other hand, in the MH algorithm, a total of $P=15$ parallel seeds were generated and each seed employed $Q=5$ Gibbs sampling processes, resulting in a total of $Q_{M H}=P \times Q \times 2 \times K=1050 \mathrm{FF}$ evaluations. Fig. 2 suggests that our HS-aided algorithm with less number of FF evaluations exhibit a lower error floor than that of the conventional MH method, which is often found at the high SNR [5]. ${ }^{1}$

Fig. 2 shows the HS-aided MUD in the context of our iterative receiver, which substantially mitigates the detrimental effect of the high correlations of random spreading sequences. It suggests that the performance improves upon increasing the number of improvisations and supporting an almost unprecedented system load as high as $\beta=6$ is possible for our HS-aided MUD, where the performance is only about $E_{b} / N_{0}=1 \mathrm{~dB}$ away from the single-user bound. At this system load, $\mathcal{Q}_{H S}=2550 \mathrm{FF}$ evaluations were used instead of $\mathcal{Q}_{\mathrm{opt}}=42 \times 2^{42}$. Although it is possible that a lower number of IDD iterations is needed when

\footnotetext{
${ }^{1}$ Proven convergence of the HS algorithm cannot be ensured, but experimental evidence shows that the distribution of the bit error ratio tends to a Dirac-like function upon increasing the affordable complexity.
} 


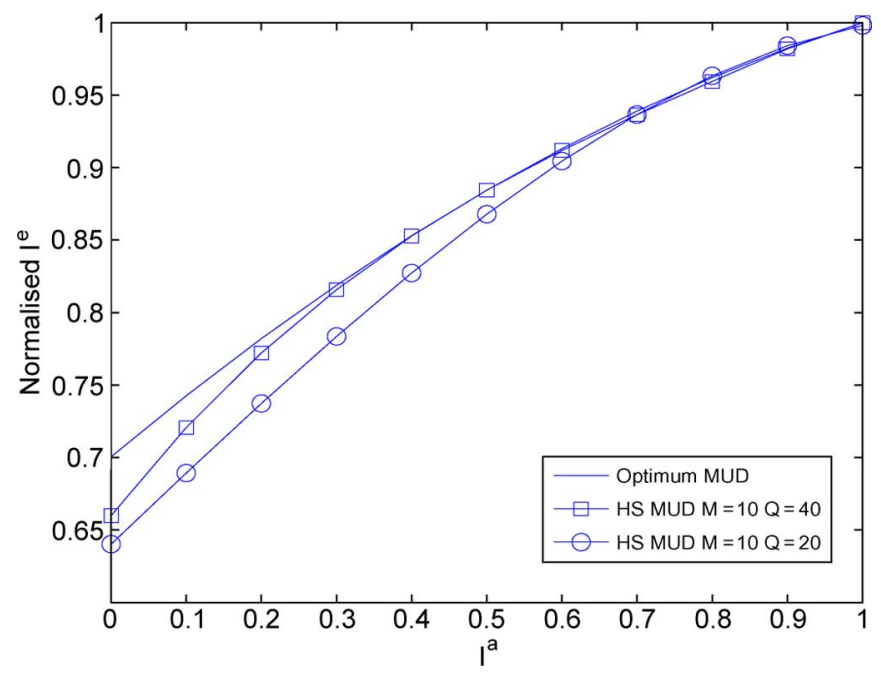

Fig. 3. Extrinsic mutual information discrepancy between the HS-aided MUD and the optimum Bayesian MUD as a function of a priori mutual information, where we set $\beta=3, N_{c}=4$ and $E_{b} / N_{0}=6 \mathrm{~dB}$. The results are normalised to the maximum mutual information value observed for the perfect a priori information $I^{a}=1$ employing the optimum MUD.

employing the optimum full-search-based MUD, when we take into account the number of iterations, the total number of FF evaluations for the HS-aided MUD becomes $\mathcal{Q}_{H S} \times I_{\mathrm{IDD}}=$ 25500 , which is still only a fraction of $\mathcal{Q}_{\text {opt }}$ even for a single iteration, when the optimum MUD is employed. We also note that the HS parameters were kept the same for both $\beta=6$ and $\beta=4$, which implies that proposed HS-aided algorithm is capable of achieving a near-single-user performance without the excessive complexity of the optimum detector and that within limits, the complexity of the algorithm is reasonably independent of $\beta$.

Fig. 3 shows the extrinsic mutual information discrepancy between the HS-aided MUD and the optimum Bayesian MUD of (1) as a function of the a priori mutual information, where we set $\beta=3, N_{c}=4$ and $E_{b} / N_{0}=6 \mathrm{~dB}$. The results are normalised to the maximum mutual information value observed for the perfect $a$ priori information of $I^{a}=1$ employing the optimum MUD. It can be seen that by increasing the number of improvisations $Q$, our HS-aided MUD becomes capable of approaching the performance of the optimum full-search-based MUD algorithm and the discrepancy becomes smaller upon increasing the amount of a priori mutual information, namely upon increasing the number of iterations within the IDD.

\section{Comments}

In comparison to GA [3], which require the tuning of a range of parameters, as long as the initial HMM size of $M$ is sufficiently large, our designed HS-aided algorithm requires the tuning of a single parameter, namely of the number of improvisations $Q$. This property is acquired as a benefit of the marginal APP-based pitch adjustment step, which equips it with the capability of attaining convergence from a Bayesian inference point of view [3]. At the same time, the entire search space is visited by the HS MUD with the aid of the randomly generated base vector from the set of $M$ rather diverse candidate vectors at each improvisation. ${ }^{2}$

The marginal APP-based pitch adjustment probability may be compared to the classic Gibbs sampling of the MH method [3]. In contrast to the successive correlated sample vectors generated by Gibbs sampling, our proposed HS algorithm does not form a correlated Markov Chain (MC), since each new harmony vector may be accepted or rejected based on the value of the FF after each improvisation. Hence, the random-walk behaviour of the $\mathrm{MH}$ method may be avoided. This is the fundamental difference in comparison to the MC based algorithms, such as [5]. On the other hand, our HS algorithm is also different from the traditional importance sampling or rejection sampling technique [3], which requires a so-called proposal distribution and is only effective in low-dimensional problems.

Remarks: Being distinct from both the GA and MH method, our HS-aided MUD algorithm may also be considered as combining the wisdom of these two classic algorithms, where the individual's modifications are generated based on the $\mathrm{MH}$ method, while crossovers are mimicked by randomly generating the base vector.

\section{CONCLUSION}

In this letter, we proposed a novel HS-aided iterative MUD and soft-decision channel decoding for DS-CDMA systems. We used the marginal APP based soft information as a variant of the pitch adjustment probability in the original HS-aided algorithm, which led to a low-complexity MUD approaching the singleuser performance even for DS-CDMA systems supporting a normalised system load of $\beta=6$. The comparison of diverse detectors in various scenarios will be our future work.

\section{ACKNOWLEDGMENT}

Fully detailed technical reports on this research are available to Industrial Members of Mobile VCE.

\section{REFERENCES}

[1] J. Boutros and G. Caire, "Iterative multiuser decoding: Unified framework and asymptotic performance analysis," IEEE Trans. Inform. Theory, vol. 48, no. 7, pp. 1772-1793, Jul. 2002.

[2] K. Yen and L. Hanzo, "Genetic algorithm assisted joint multiuser symbol detection and fading channel estimation for synchronous CDMA systems," IEEE J. Sel. Areas Commun., vol. 19, no. 6, pp. 985-998, Jun. 2001.

[3] D. J. MacKay, Information Theory, Inference and Learning Algorithms. Cambridge, U.K.: Cambridge Univ. Press, 2003.

[4] Z. W. Geem, Music-Inspired Harmony Search Algorithms: Theory and Applications. Berlin, Germany: Springer-Verlag, 2009.

[5] B. Farhang-Boroujeny, H. Zhu, and Z. Shi, "Markov Chain Monte Carlo algorithms for CDMA and MIMO communication systems," IEEE Trans. Signal Process., vol. 54, no. 5, pp. 1896-1909, May 2006.

\footnotetext{
${ }^{2} \mathrm{~A}$ small HMM contains an insufficient number of harmony vectors, which limits the exploration capability of the search algorithm, since the diversity of the associated samples is reduced. This will lead to a faster initial-converge. Too large a HMM requires many FF evaluations and it does not necessarily lead to a performance improvement, unless - by coincidence-the optimum candidate solution happens to be part of the search pool, which is unlikely when considering a high-dimensional problem.
} 